ACCEPTED MANUSCRIPT

\title{
Glassy Carbon MEMS for Novel Origami-Styled 3D Integrated Intracortical and Epicortical Neural Probes
}

To cite this article before publication: Noah Goshi et al $2018 \mathrm{~J}$. Micromech. Microeng. in press https://doi.org/10.1088/1361-6439/aab061

\section{Manuscript version: Accepted Manuscript}

Accepted Manuscript is "the version of the article accepted for publication including all changes made as a result of the peer review process, and which may also include the addition to the article by IOP Publishing of a header, an article ID, a cover sheet and/or an 'Accepted Manuscript' watermark, but excluding any other editing, typesetting or other changes made by IOP Publishing and/or its licensors"

This Accepted Manuscript is @ 2018 IOP Publishing Ltd.

During the embargo period (the 12 month period from the publication of the Version of Record of this article), the Accepted Manuscript is fully protected by copyright and cannot be reused or reposted elsewhere.

As the Version of Record of this article is going to be / has been published on a subscription basis, this Accepted Manuscript is available for reuse under a CC BY-NC-ND 3.0 licence after the 12 month embargo period.

After the embargo period, everyone is permitted to use copy and redistribute this article for non-commercial purposes only, provided that they adhere to all the terms of the licence https://creativecommons.org/licences/by-nc-nd/3.0

Although reasonable endeavours have been taken to obtain all necessary permissions from third parties to include their copyrighted content within this article, their full citation and copyright line may not be present in this Accepted Manuscript version. Before using any content from this article, please refer to the Version of Record on IOPscience once published for full citation and copyright details, as permissions will likely be required. All third party content is fully copyright protected, unless specifically stated otherwise in the figure caption in the Version of Record.

View the article online for updates and enhancements. 


\section{Glassy Carbon MEMS for Novel Origami-Styled 3D Integrated Intracortical and Epicortical Neural Probes}

Noah Goshi ${ }^{\mathrm{a}}$, Elisa Castagnola ${ }^{\mathrm{a}, \mathrm{b}}$, Maria Vomero ${ }^{\mathrm{c}}$, Calogero Gueli ${ }^{\mathrm{c}}$, Claudia Cea ${ }^{\mathrm{a}, \mathrm{b}}$, Elena Zucchini $^{\mathrm{d}}$, David Bjanes ${ }^{\mathrm{b}, \mathrm{e}}$, Emma Maggiolini ${ }^{\mathrm{d}}$, Chet Moritz ${ }^{\mathrm{b}, \mathrm{f}}$, Sam Kassegne ${ }^{\mathrm{a}, \mathrm{b}}$, Davide Ricci ${ }^{\mathrm{d}}$, Luciano Fadiga ${ }^{\text {d,g }}$

a. MEMS Research Lab., Department of Mechanical Engineering, College of Engineering, San Diego State University, 5500 Campanile Drive, San Diego, CA 92182-1323, USA

b. Center for Sensorimotor Neural Engineering (CSNE), Box 37, 1414 NE 42nd St., Suite 204, Seattle, WA 98105-6271, USA

c. Laboratory for Biomedical Microsystems, Institute for Microsystem Technology (IMTEK), University of Freiburg, Georges-Köhler-Allee 103, 79110 Freiburg im Breisgau, Germany

d. Center for Translational Neurophysiology of Speech and Communication, Istituto Italiano di Tecnologia, Via Fossato di Mortara 17/19, 44121, Ferrara, Italy

e. University of Washington, Department of Electrical Engineering, Seattle, WA

f. University of Washington, Division of Physical Therapy, Departments of Rehabilitation Medicine and Physiology and Biophysics, Seattle, WA

g. Section of Human Physiology, University of Ferrara, Via Fossato di Mortara 17/19, 44121, Ferrara, Italy

* Previously at MEMS Research Lab., Department of Mechanical Engineering, College of Engineering, San Diego State University, 5500 Campanile Drive, San Diego, CA 92182-1323, USA

e-mail address of each author: nkgoshi@ucdavis.edu ecastagnola@sdsu.edu maria.vomero@imtek.uni-freiburg.de claudia.cea90@gmail.com gerozte@gmail.com elena.zucchini@iit.it davidbjanes@gmail.com emma.maggiolini@iit.it ctmoritz@uw.edu kassegne@mail.sdsu.edu davide.ricci@iit.it luciano.fadiga@iit.it Correspondence and requests for materials should be addressed to Elisa Castagnola e-mail: ecastagnola@sdsu.edu

Full postal address: MEMS Research Lab., EIS Building, Department of Mechanical Engineering, College of Engineering, San Diego State University, 5500 Campanile Drive, San Diego, CA 921821323, USA. 


\begin{abstract}
We report on a novel technology for microfabricating 3D origami-styled Micro Electro-Mechanical Systems (MEMS) structures with glassy carbon (GC) features and a supporting polymer substrate. GC MEMS devices that open to form 3D microstructures are microfabricated from GC patterns that are made through pyrolysis of polymer precursors on high-temperature resisting substrates like silicon or quartz and then transferring the patterned devices to a flexible substrate like polyimide followed by deposition of an insulation layer. The devices on flexible substrate are then folded into 3D form in an origami-fashion.

These 3D MEMS devices have tunable mechanical properties that are achieved by selectively varying the thickness of the polymeric substrate and insulation layers at any desired location. This technology opens new possibilities by enabling microfabrication of a variety of 3D GC MEMS structures suited to applications ranging from biochemical sensing to implantable microelectrode arrays. As a demonstration of the technology, a neural signal recording microelectrode array platform that integrates both surface (cortical) and depth (intracortical) GC microelectrodes onto a single flexible thin-film device is introduced.
\end{abstract}

When the device is unfurled, a pre-shaped shank of polyimide automatically comes off the substrate and forms the penetrating part of the device in a 3D fashion. With the advantage of being highly reproducible and batch-fabricated, the device introduced here allows for simultaneous recording of electrophysiological signals from both the brain surface (electrocorticography - ECoG) and depth (single neuron). Our device, therefore, has the potential to elucidate the roles of underlying neurons on the different components of $\mu$ ECoG signals. For in-vivo validation of the design capabilities, the recording sites are coated with a poly(3,4-ethylenedioxythiophene) - polystyrene sulfonate - carbon nanotube composite, to improve the electrical conductivity of the electrodes and consequently the quality of the recorded signals. Results show that both $\mu \mathrm{ECoG}$ and intracortical arrays were able to acquire neural signals with high-sensitivity that increased with depth, thereby verifying the device functionality. 
Keywords: MEMS, Origami Structures, Microelectrode array (MEA), Thin-film technology, $\mu$ ECoG, intracortical electrode, Glassy Carbon, PEDOT-PSS-CNT coating.

\section{Introduction}

Carbon, as a technology material, is finding a renewed research and industrial interest. As evidenced by the continued interest in graphene and other forms of carbon such as fullerene, carbon nanotubes (CNT), carbon nanofibers, carbon nano-composites, etc., carbon is poised to become a critical technology material with wide application in a variety of disciplines [1-3]. In addition to the traditional areas where the superior electrical and electrochemical properties of carbon have made it a material of choice, recently a renewed focus to this material has been brought by bioengineering research aimed to electrically interfacing it with biological tissues. In turn, these factors have highlighted the need for innovation in robust and versatile micro and nanofabrication technology of carbon-based devices to meet this increasing demand. In response to that, the field of glassy carbon MEMS (GC MEMS), which involves the patterning of a photosensitive polymer precursor on a silicon substrate followed by pyrolysis of the polymer layer to convert it to glassy carbon, has gained traction and increased range of applications [4-6].

Further, the recent introduction of a new pattern transfer technology that allowed GC structures to be supported on polymeric substrates as opposed to only high-temperature resistant silicon and quartz, has enabled GC MEMS technology to enter a new phase [6-8]. This new pattern transfer technology has been demonstrated through the microfabrication of a glassy carbon-based array of microelectrodes on a flexible substrate for applications in neural sensing and stimulation, particularly for $\mu \mathrm{ECoG}$ (Micro-Electrocorticography) systems. While innovation in this area is expected to continue, the next set of challenges in expanding the application of GC MEMS involve the ability to build complex 3D origami-style structures using carbon as electrical and mechanical component, an approach similar to earlier 3D microprobes microfabricated using standard metal fabrication processes [9]. The origami-type technology presented in this research addresses this need and consists of microfabricating GC structures on high-temperature resisting substrates like 
silicon, quartz or similar materials and then transferring the patterned devices on to a flexible substrate like polyimide, and finally adding photolithographically patternable 2D layers that will open up to form 3D structures. It has to be noted that while there are other reports of out-of-plane, foldable MEMS devices, they often involve fabrication steps that are incompatible with the high temperatures required for the pyrolysis of GC [10] and require stiff substrates [11]. Therefore, these reported probes are not suitable for implantable neural electrodes and involve extremely precise and time consuming manual manipulation $[12,13]$.

As a demonstration of this new technology, we present here a neural probe made of a microelectrode array platform that integrates both surface $(\mu \mathrm{ECoG})$ and penetrating (intracortical) GC electrodes onto a single flexible thin-film device. This is a natural extension, as GC has been demonstrated to be a compelling platform for brain-computer interface (BCI) applications due to its superior electrical and electrochemical properties and excellent surface chemistry that enables enhanced adhesion to conductive polymers [8]. Along these lines, understanding how the brain translates individual neural signals into behaviors, motivation and actions, requires the recording of electrophysiological signals from large populations of neurons over multiple regions of the brain [14]. While implantable microwires [15], tetrodes [16] and multiple electrode arrays (MEAs) [17] are capable of sampling single-unit action potentials with high spatial and temporal resolution, the implantation of the number of electrodes required to monitor such a large population of neurons may not be feasible due to the possible damage to the brain tissue during implantation [18]. On the other hand, ECoG arrays are capable of sampling from large neural populations as they are placed on the surface of the brain, but do not provide the same spatial resolution as penetrating electrodes [19]. MEMS fabrication technologies have been applied to ECoG arrays to address these shortcomings and have led to improved data acquisition through an increase in electrode density [20-22], improved electrode materials and fabrication techniques [22-24], and recordings of single unit action potentials from subdural ECoG arrays [25]. Following these improvements, ECoG arrays are becoming a platform of choice for BCI studies, mainly due to their ability to address 
large areas of the cortex and support chronic implants owing to their reduced invasiveness [26-28]. Furthermore, these improvements in ECoG technology have motivated the further investigation of the relationship between signals recorded from the brain surface and those generated by neurons located at different levels of the cortex to fully exploit the potential of the ECoG platform. In fact, research has already indicated a strong correlation between surface signals and the activity of underlying neurons in the visual [29] and barrel [30] cortex in rats and in the primary somatosensory [31] and primary motor [32] cortex in non-human primates.

Verification of this correlation between surface signals and the activity of the underlying neurons requires the simultaneous recording of surface and intracortical signals. However, while there has been significant research into ECoG array technology and in understanding the relationship between ECoG signals and signals from underlying neurơns, improving the technology to simultaneously record these signals has been largelyleft unaddressed. Nearly all the aforementioned electrophysiology experiments use separate systems to record the different populations of neurons. Early experiments used single ball electrodes for ECoG recordings in conjunction with a penetrating tetrode array [31]. Later, specialized ECoG arrays were developed with multiple holes in the supporting substrate that allow the insertion of intracortical electrode arrays through the ECoG device [29-32]; however, this approach still required fabrication, placement and connection of multiple electrode systems. Some groups have developed integrated electrode platforms to address these needs using either epoxy fixation of an intracortical MEA through an ECoG array [29] or through specialized fabrication techniques to form SU8 microneedles on the surface of a thin-film electrode array [33-34]. However, both designs required specialized fabrication techniques, with the former demanding that each device be individually assembled, and thereby dramatically increasing both the fabrication time and variability between the assembled devices. Furthermore, these designs introduce a new limitation, in that the penetration depth of the intracortical MEA is set during device fabrication and assembly and, therefore, cannot be adjusted during the implant surgery. 
We submit that the new thin-film platform presented here, that combines both intracortical and surface GC electrodes into a single device through origami-like unfolding of the substrate to 3D structure, addresses these needs. Such device can be batch-fabricated using recently developed pattern-transfer techniques [6] to incorporate GC electrodes, and has the advantage of being completely self-assembled. The device was initially fabricated in a 2D geometry that takes the final 3D from, when it is folded into a zero-insertion force (ZIF) connector. In this report, we demonstrate the ability to fabricate $3 \mathrm{D}$ platforms with varying geometries and multiple penetrating arrays. We also verify the ability of the traces to maintain their connectivity when folded into 3D form by electrochemical characterization. To verify in vivo performance of our platform, we fabricated a device consisting of single penetrating array with several microelectrodes flanked by two $\mu \mathrm{ECoG}$ arrays with a spring like system that allows the intracortical array to be inserted to varying depths without change to the $\mu \mathrm{ECoG}$ electrode placements. As PEDOT coatings are useful to guarantee low impedance and ideal electrode conductivity of miniaturized electrodes [33], we coated the $30 \mu \mathrm{m}$ diameter GC microelectrodes with PEDOT-PSS, reinforced with CNTs, which further improve mechanical and electrical properties [35-39]. Following fabrication, we performed an in vivo validation of the device by recording neural activity from the vibrissae representation of the rat primary somatosensory cortex $(\mathrm{S} 1)$, namely the barrel cortex, leveraging the well-known cytoarchitecture and the physiology of this region [40-43].

\section{Materials and Methods}

In this section, we discuss the design of origami-style polymeric substrate supported GC MEMS neural probes, their microfabrication, and characterizations.

\subsection{Microfabrication GC Microelectrode Arrays}

The basic core fabrication method for the pattern transfer of a GC microelectrode array on a polyimide substrate is described in detail elsewhere [6-8]. Here, we extend the technology by adding a new reinforcing layer that is selectively deposited and patterned at areas of interest that allow a 3D origami-style unfurling. This modified process is summarized in Figure 1. 
The microfabrication process starts with spin-coating SU8 negative photoresist (Microchem, MA) at $1000 \mathrm{rpm}$ for $60 \mathrm{sec}$ and soft-baking at $65^{\circ} \mathrm{C}$ for $10 \mathrm{~min}$ and $95^{\circ} \mathrm{C}$ for $30 \mathrm{~min}$ followed by $\mathrm{UV}$ exposure at $\sim 400 \mathrm{~mJ} / \mathrm{cm}^{2}$. Post-exposure bake consists of $65^{\circ} \mathrm{C}$ for $1 \mathrm{~min}$ and $95^{\circ} \mathrm{C}$ for $10 \mathrm{~min}$. This was followed by development of SU8 for 3-5 mins and curing at $150^{\circ} \mathrm{C}$ for 30 mins. Pyrolysis $\left(1000^{\circ} \mathrm{C}\right.$ in an inert $\mathrm{N}_{2}$ environment) of the negative resist layer follows protocols described elsewhere [6-8]. After pyrolysis step, $6 \mu \mathrm{m}$ layer of photo-patternable polyimide (HD 4100) (HD Microsystems) was spin-coated on top of GC microelectrodes at $2500 \mathrm{rpms}$ for 45 seconds, soft baked at $90^{\circ} \mathrm{C}$ for $3 \mathrm{~min}$ and at $120^{\circ} \mathrm{C}$ for $3 \mathrm{~min}$, then cooled down to room temperature, and patterned through UV exposure at $\sim 400 \mathrm{~mJ} / \mathrm{cm}^{2}$. Post-exposure bake consisted of $80^{\circ} \mathrm{C}$ for $1 \mathrm{~min}$. Development was performed using a spry-puddle process where QZ3501 (Fuji Film) was dispersed to form a puddle on a stationary wafer. Rinse was applied after a set time of $15 \mathrm{sec}$, followed by spin-drying of the wafer (2000 rpms for $15 \mathrm{sec}$ and $500 \mathrm{rpm} / \mathrm{s}$ ramp). The cycle was repeated 3 times and then the wafer was rinsed with SU8 developer (MicroChem). Subsequently, the polyimide layer was partial cured at $300^{\circ} \mathrm{C}$ for 60 mins under $\mathrm{N}_{2}$ environment.

Following this, metal traces were deposited using NR9-1000PY negative photoresist (Futurrex) as a sacrificial layer. Futurrex was spin coated at 500 rpms for 45 seconds and ramped down for 10 seconds, then prebaked for $2 \mathrm{~min}$ at $150^{\circ} \mathrm{C}$ followed by $380 \mathrm{~mJ} / \mathrm{cm}^{2} \mathrm{UV}$ exposure. Post exposure bake was done at $100^{\circ} \mathrm{C}$ for 2 minutes and sample was developed in pure RD6 developer (Futurrex Inc.) for 3 seconds. Subsequently, $20 \mathrm{~nm}$ chromium adhesion layer and $200 \mathrm{~nm}$ gold layer were deposited through sputtering. After metal deposition, a lift-off process was performed and the sacrificial layer was removed in acetone. To electrically insulate the device, $6 \mu \mathrm{m}$ of polyimide HD4100 (300 rpms) was spun, patterned $\left(400 \mathrm{~mJ} / \mathrm{cm}^{2}\right)$ and cured $\left(350^{\circ} \mathrm{C}\right.$ for 90 mins under $\mathrm{N}_{2}$ environment). An additional thicker layer $(30 \mu \mathrm{m})$ of polyimide (Durimide 7520$)$ (Fuji Film) that eventually becomes 3D structure was spin-coated $(800 \mathrm{rpm}, 45 \mathrm{~s})$ and then patterned $\left(400 \mathrm{~mJ} / \mathrm{cm}^{2}\right)$ on top of the insulation layer to selectively reinforce the penetrating portion of the device. Then it was developed, as previously described, and final cured at $350^{\circ} \mathrm{C}$ for 90 minutes. 
Subsequently, the device was released from the wafer through selective etching of silicon dioxide with buffered hydrofluoric acid. The device was assembled into the final 3D form and held in place by inserting both ends of the device into a custom PCB that served as the connector to the electrophysiological recording system. Representative images of devices after being released from the carrying wafer in their 2D form and of the origami-styled unfurled device assembled into connecting PCB in their 3D conformation are shown in Figures 2 and 4.

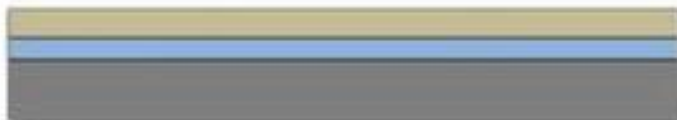

a.

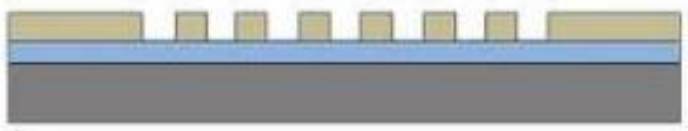

b.

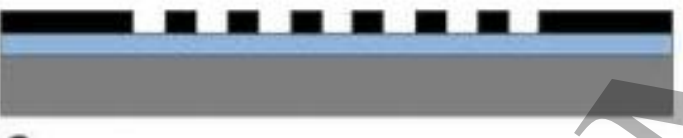

C.

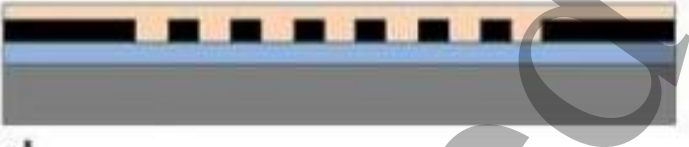

d.

- Si $\square \mathrm{SiO}_{2} \square \mathrm{SU}-8 \square \mathrm{GC}$

Au (with $\mathrm{Cr}$ adhesion layer) e.
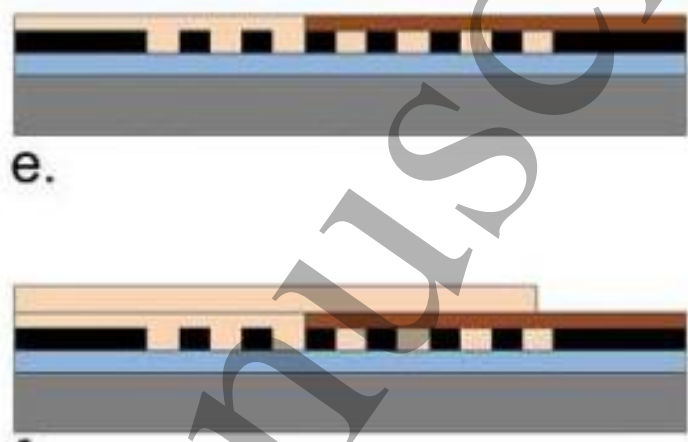

f.

g.

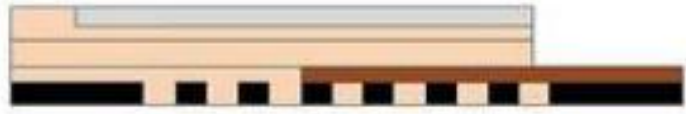

h.

\section{Polyimide (folding)}

Figure 1. MEMS process for fabricating the multi-layered thin-film GC electrodes: a. Spin-coat negative photoresist (SU-8); b. Pattern SU-8 through lithography; c. Pyrolysis; d. Spin-coat and pattern polyimide base layer; e. Metal deposition and lift-off for traces and bump-pads; f. Spin-coat and pattern the polyimide insulation layer to get bump-pads access; g. Spin-coat and pattern the polyimide folding layer (Durimide 7520); h. Etch $\mathrm{SiO}_{2}$ with $\mathrm{BOE}$ to release device from wafer. 


\subsection{Design of GC MEMS Origami Structures - Example of 3D GC Microelectrode Array}

To demonstrate the versatility of the new architecture and modified pattern transfer technology introduced here, we highlight the device design of a portfolio of origami-type 3D probe structures. The devices were initially designed in a 2D geometry that could be batch-fabricated on a carrying wafer and when released, folded, and inserted into a ZIF connector would take on 3D form (Figure 2 and 3). The 2D geometry consisted of a series of cut-outs that, when folded, allowed the penetrating portions of the device to remain "in-plane," while the folded areas formed the ECoG portion of the device. Figure 2 shows a portfolio of such devices with (i) single or multiple shanks that unfurl from a 2D configuration to a 3D form and (ii) flat and foldable flaps that support the structure and contain pads for electrical connections. The combination of such geometries allows probes to assume a wide variety of conformations, and hence applications. It should be stressed that the flaps that contain surface microelectrodes were patterned from polyimide (HD4100) whereas the shanks that form penetrating microelectrode array were typically patterned from thicker polyimide, Durimide 7520 .

Figure 3 shows a schematic of the device folding. Due to the cut-outs in the 2D geometry, when the device is folded upon itself, the penetrating portion of the device does not experience this backward force, and therefore remains in-plane, while the bulk of the device is folded back upon itself, and thereby forms the out-of-plane, 3D geometry of the ECoG portion. An additional layer of thicker polyimide (Durimide 7520) was patterned over the penetrating shanks to increase their mechanical robustness during 3D unfurling and allow penetration through the cortical tissue without the need for a penetration aid. The thickness of the layer of polyimide was determined by comparing the force required to penetrate the cerebral cortex of the rat, $\sim 1.5 \mathrm{mN}$ with the dura mater removed [44], to the critical buckling load of the penetrating shank calculated using the formula for Euler's critical load given below, $\mathrm{P}_{\mathrm{cr}}=\left(\pi^{2} \mathrm{EI}\right) /(\mathrm{KL})^{2}$

where Pcr is Euler's critical load, E is the Young's modulus of the material, I is the area moment of 
inertia, $\mathrm{K}$ is the column effective length factor and $\mathrm{L}$ is the length of the column. The critical loads for the penetrating portions of the different designs is given in Table 1 .

\begin{tabular}{llllll}
\hline Device & Width & Height & Length & Young's Modulus & Critical Load \\
\hline Figure 2 B/C & $1 \mathrm{~mm}$ & $30 \mu \mathrm{m}$ & $2.5 \mathrm{~mm}$ & $2.5 \mathrm{GPa}[45]$ & $8.8 \mathrm{mN}$ \\
\hline Figure 2 D & $1 \mathrm{~mm}$ & $30 \mu \mathrm{m}$ & $3.5 \mathrm{~mm}$ & $2.5 \mathrm{GPa}[45]$ & $4.53 \mathrm{mN}$ \\
\hline Figure 4 & $1 \mathrm{~mm}$ & $30 \mu \mathrm{m}$ & $5.7 \mathrm{~mm}$ & $2.5 \mathrm{GPa}[45]$ & $1.7 \mathrm{mN}$ \\
\hline
\end{tabular}

Table 1. Critical buckling load calculation for the penetrating portions of the different probe designs.

As the thicker layer of polyimide was only patterned over the penetrating shanks, the ECoG portion of the device would remain ultra-flexible to promote contact between the electrodes and the surface of the brain. Alternatively, a single foldable flap platform (Figure 2D) was designed for chronic implantations in free moving animals, where disconnections between the ZIF connector and the device pose a greater concern. The ultra-flexible flap containing the ECoG electrodes can be simply held away from the penetration site using tether or forceps and following the implantation of the penetrating portion be allowed to fall back into contact with the cortex.

Assessment of the biocompatibility of the specific polyimide used (Polyimide HD4100 and Durimide 7520) for implantable medical device use, along with their suitability for peripheral nerve and cortical implants and epiretinal stimulation, has been reported in earlier studies (46-51).

\subsection{Assembly of GC MEMS Origami Structure}

To validate the patterning technology, we chose to fabricate a single-shank penetrating array as seen in Figure 4 specifically designed for an acute in vivo validation study in a rat. The curved conformation the $\mu \mathrm{ECoG}$ array substrate assumes when folded into its $3 \mathrm{D}$ form acts as a spring-like structure that allows the penetrating array to be inserted at varying depths, while the parts of $\mu \mathrm{ECoG}$ array made of polyimide remains stationary. For this study, the device was designed to record from the barrel cortex in rats. The electrodes in both arrays are $30 \mu \mathrm{m}$ in diameter and have a center to center pitch of $300 \mu \mathrm{m}$ in the intracortical array and $500 \mu \mathrm{m}$ for the $\mu \mathrm{ECoG}$ array. The 
intracortical array contains five electrodes stacked vertically, with the deepest electrode reaching a depth at least $2.3 \mathrm{~mm}$. A detailed schematic of the device, reporting the overall dimensions, diameter of the electrodes and center to center distance is provided in Supplementary Figure 1. This penetration depth and electrode spacing allows the device to record signals from at least one electrode for each cortical layer (from layer II to layer V). The $\mu \mathrm{ECoG}$ array contains additional six microelectrodes (three on each side of the intracortical array) to allow comparison of surface recordings at different locations with depth recordings through the cortex.

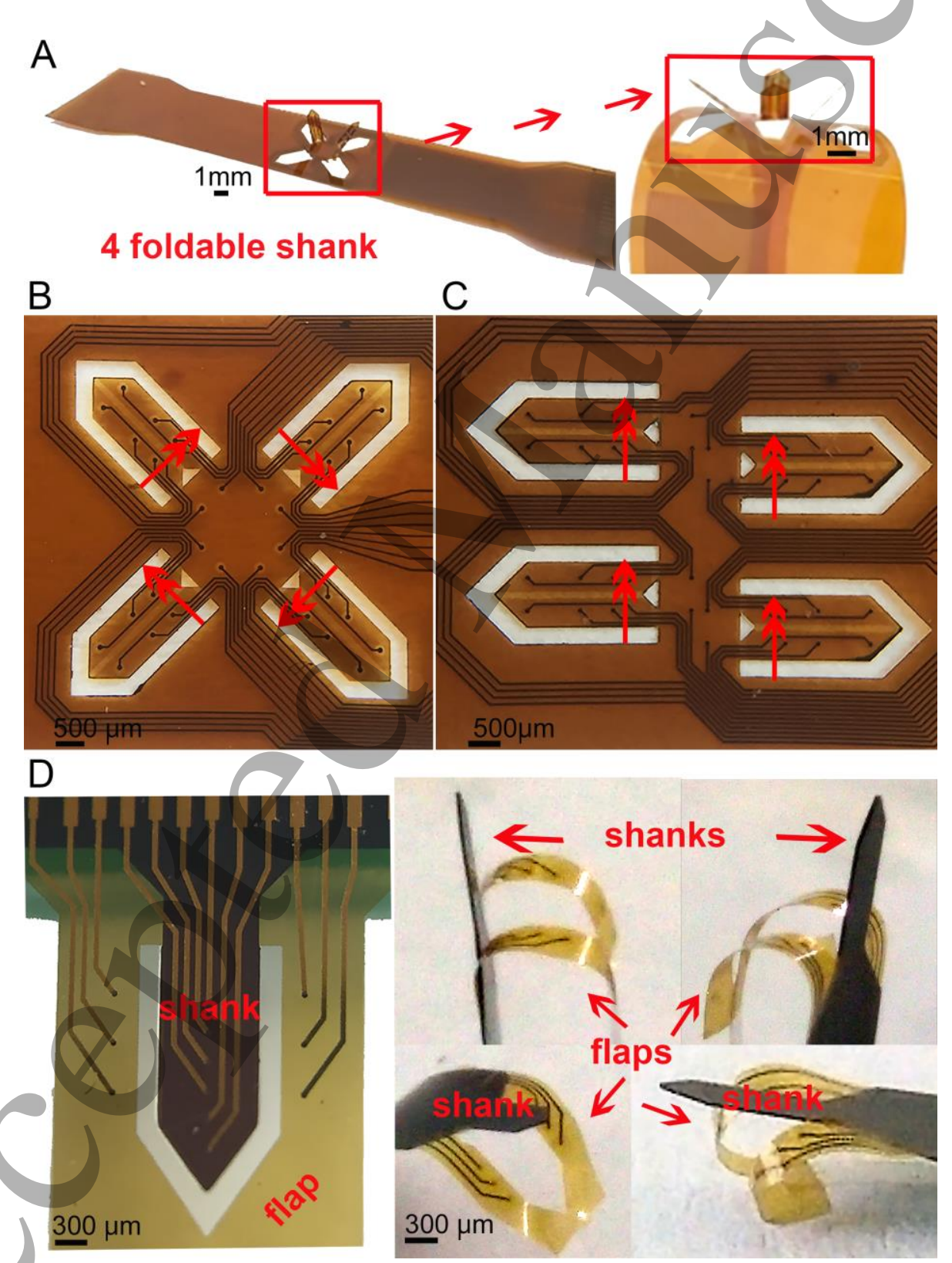

Figure 2. Origami MEMS Portfolio. (A) and (B) Four-shanks origami probes. (B) and (C) Magnifications of two possible probe designs (D) Single-shank origami probe with foldable flap. 


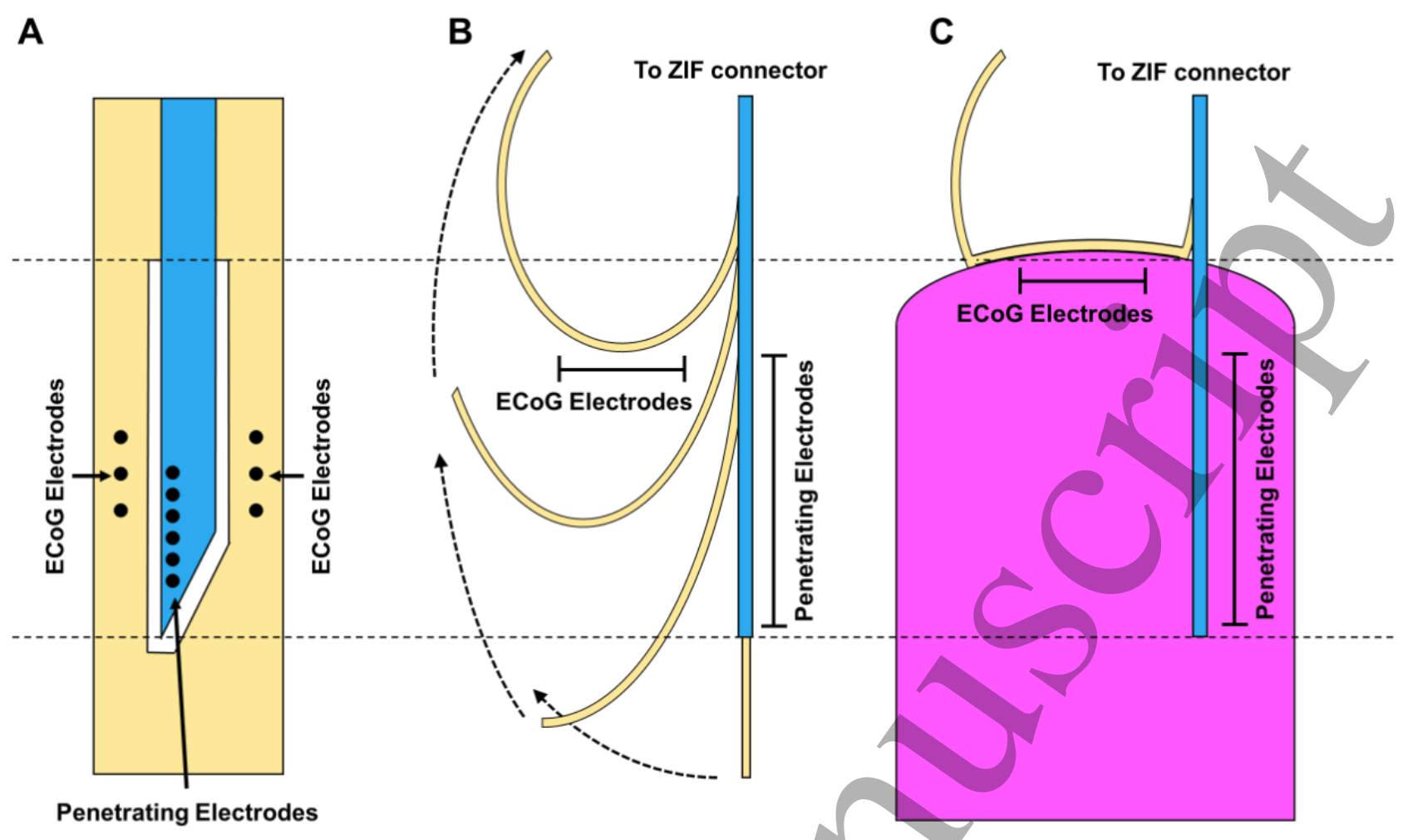

Figure 3. Schematic of the folding process and implantation of a 3D integrated intracortical and epicortical neural probe. (A) Overhead schematic of the device immediately following release from the wafer. (B) Schematic of the device folding to the final 3D form by the insertion of both ends into the ZIF connector. Due to the 2D geometry of the device, the penetrating portion is not subjected to the force back towards the ZIF connector, and therefore remains in-plane, while the ECoG electrodes are folded back towards the connector creating the 3D structure. (C) Schematic of the device implanted in the cortex of a rat. Due to the ultra-flexibility of the ECoG portion, the device is able to follow the surface of the cortex, promoting contact between the electrodes and the surface of the brain. The probe is able to bend through almost $180^{\circ}$ angle, if needed.

\subsection{PEDOT-PSS-CNT electrochemical deposition}

Poly(3,4-ethylenedioxythiophene) (PEDOT) and carboxylated MWCNTs (COOH-CNTs, NC 3151, $<4 \%$ of $\mathrm{COOH}$ functional groups, Nanocyl S.A., Belgium) nanocomposites (PEDOT-CNT) were co-electrodeposited from a 0.5 M 3,4-ethylenedioxythiophene (EDOT, Sigma-Aldrich, USA) aqueous solution containing $1 \mathrm{mg} / \mathrm{ml}$ of suspended $\mathrm{COOH}-\mathrm{CNT}$ and $0.6 \mathrm{wt} \%$ of poly(sodium 4styrenesulfonate) (PSS) (Sigma-Aldrich, USA). COOH-CNTs were suspended in ultrapure water 
(Milli-Q, Millipore, USA) by horn sonication (Vibra-Cell VCX130, Sonics and Materials, USA) (6 $\mathrm{s}$ at $66 \%$ duty cycle pulses, $4 \mathrm{~W} / \mathrm{ml}$ ) for 30 min while keeping the solution cooled with an ice bath. PSS and the monomer were added to the suspension immediately afterwards, and the solution was kept deoxygenated by bubbling nitrogen. The electrochemical deposition was carried out using a polymerization potential of $0.8 \mathrm{~V}$ vs. reference electrode for $10 \mathrm{CV}$ cycles. Depositions were carried out using a potentiostat/galvanostat (PARSTAT 2273, Princeton Applied Research, USA) connected to a three-electrode electrochemical cell with a platinum counter electrode and an $\mathrm{Ag} / \mathrm{AgCl}$ reference electrode.

\subsection{Device Characterization}

Once the devices were assembled in 3D fashion, the electrochemical characterization of the devices was carried out in $0.9 \% \mathrm{NaCl}$ aqueous solution through electrochemical impedance spectroscopy (EIS). EIS measurements were performed by superimposing a sine wave (10 mV RMS amplitude) onto the open circuit potential, in the frequency range from 1 to $10^{5} \mathrm{~Hz}$. EIS were carried out using a potentiostat/galvanostat (Reference 600, Gamry Instruments, USA) connected to a three-electrode electrochemical cell with a Pt counter electrode and a $\mathrm{Ag}|\mathrm{AgCl}| \mathrm{KCl}$ (sat.) reference electrode.

The capacitive charging ability of the PEDOT-PSS-CNT coated GC microelectrodes was evaluated by cyclic voltammetry (CV) and voltage transient in a physiological aqueous solution $(\mathrm{NaCl} 0.9 \%)$. For CVs, the potential on the working electrode was swept between 0.8 and $-0.6 \mathrm{~V}$ vs $\mathrm{Ag} / \mathrm{AgCl}$ at a scan rate of $100 \mathrm{mV} / \mathrm{s}$, starting at open-circuit potential and sweeping in the positive direction first. The Charge Transfer Capacities (CTC) were calculated as the time-integral of an entire CV cycle between 0.5 and $-0.5 \mathrm{~V}$, or between -0.6 and $0.8 \mathrm{~V}\left(\mathrm{CTC}_{2}\right)$. For voltage transient, a cathodic-first charge-balanced biphasic symmetric current pulse with $400 \mu$ s cathodic half-phase period was used. The charge injection limit, defined as the maximum quantity of charge an electrode can inject before reaching the water electrolysis potential, was calculated as the time integral of the current in the loading phase normalized by the geometric area of the microelectrode. 


\subsection{In-vivo Test}

The device with GC microelectrodes coated with PEDOT-PSS-CNT was in-vivo validated. The surgical procedures were performed on one Long-Evans male rat (weight 400g). The experimental plan was designed in compliance with the guidelines established by the European Communities Council (Directive 2010/63/EU, Italian Legislative Decree n. 26, 4/3/2014) and the protocol was approved by the Ethics Committee for animal research of the University of Ferrara and by the Italian Ministry of Health (authorization $n^{\circ} 332 / 2015-P R$ ). The animal was anesthetized with a mixture of Zoletil $(30 \mathrm{mg} / \mathrm{kg})$ and Xylazine $(5 \mathrm{mg} / \mathrm{kg})$ delivered intraperitoneally. The depth of anesthesia was monitored for the entire duration of the experimental session by testing the absence of hindlimb withdrawal reflex and was maintained by additional doses of anesthetic (i.m.). Under anesthesia, the animal was placed on stereotaxic apparatus (David Kopf Instruments, USA) and an incision of $2 \mathrm{~cm}$ was made along the midline of the cranium. The underlying muscle and connective tissue were retracted to expose the skull and a craniotomy of $5 \times 5 \mathrm{~mm}$ was made on the parietal bone to expose the primary somatosensory cortex (S1) [52].

To record the intracortical neural activity, the shaft was lowered into the vibrissa region of S1 (A-P coordinates between -1.5 and $-5.0 \mathrm{~mm}$ and M-L coordinates between 3.5 and $5.5 \mathrm{~mm}$ ) at the maximum depth of $2 \mathrm{~mm}$ to enable the 5 penetrating electrodes acquire signals from the different layers of the cortex. To test the recording capability of the 6 surface electrodes, somatosensory evoked potentials (SEP) were elicited through mechanical whiskers stimulation (see below). Eight $25 \mathrm{~min}$ sessions of evoked activity and two $10 \mathrm{~min}$ of spontaneous activity were recorded. To elicit neural activity, a vibrating system was used to produce a multi-whiskers deflection along the horizontal plane. Three different mechanical stimulation patterns were used. The whiskers, contralateral to the recorded cortex, were cut $1 \mathrm{~cm}$ from the base and included in a Velcro strip attached to a rod moved by a shaker (Type 4810 mini shaker; Bruel \& Kjaer) controlled by a National Instruments board (Austin, TX, USA). The deflection stimulus consisted of 10 trains of 10 truncated Gaussians of $12 \mathrm{~ms}$ duration at $9 \mathrm{~Hz}$ followed by a $5 \mathrm{sec}$ pause. The amplitude of the 
stimulus was changed after each train to obtain multi-whiskers deflections respectively of 250,500 , and $800 \mu \mathrm{m}$. Each train of the different amplitude was repeated 20 times with 60 seconds pause between cycles.

Electrophysiological data were acquired using a TDT multi-channel recording system 3 (Tucker Davis Technologies, USA) including ZIF-Clip ${ }^{\circledR}$ head-stage with unity (1x) gain, RZ2 real-time processor and PZ2-256 battery-powered preamplifier. Data was digitized at a sample rate of 24,414 samples/s (18-bit resolution) and transferred from RZ2 to the PC by fast fiber optic connection. Evoked and spontaneous activity data were thus stored on a PC for the subsequent offline analysis. To analyze the recording performance of the 5 penetrating electrodes, the signal-to-noise ratio (SNR) was computed on the high frequency components of the signal. SNR was calculated as peakto-peak amplitude of the mean waveform detected, divided by twice the standard deviation $(\sigma)$ of the noise.

For each penetration, the signal was digitally high-pass filtered (Butterworth, 4-poles) above 250 $\mathrm{Hz}$ and the waveform discrimination was performed at a voltage threshold of 2 times the root-mean square voltage of the background noise. The signal amplitude was taken as the mean peak-to-peak amplitude of the waveform detected and the $\sigma$ Noise was the standard deviation of the data stream after all waveforms have been removed. The Off-Line Sorter software (Plexon Inc, USA) on the continuous traces was used.

To extract the SEPs from the surface, the signal was down-sampled at $3051.8 \mathrm{~Hz}$ and digitally lowpass filtered (Butterworth 2nd order) below $300 \mathrm{~Hz}$ using MATLAB software (Mathworks, USA). The evoked responses for each amplitude of whiskers deflection were averaged from 0 to $100 \mathrm{~ms}$ after trigger pulses using EEGLAB MATLAB toolbox and OriginPro (OriginLab, USA).

\section{Results}

\subsection{GC MEMS Origami Structure}

We were able to design and fabricate a platform that incorporated GC electrodes into a 3D thin-film flexible substrate that is capable of recording both epicortical and intracortical electrophysiological 
signals in a robust and reproducible manner. Additionally, the platform is compatible with a number of different device geometries and can be tailored to the electrophysiological study of particular interest. Folding and assembly of the different devices was accomplished in the same step and simply involved inserting both ends of the device into the ZIF clip of the PCB (Figure 4B). The two ends of the device were designed to fit snugly into the connector $(<200 \mu \mathrm{m}$ difference between the width of the device and connector) and the device folding could be done quickly and repeatedly. Alternatively, for devices with a smaller footprint (Figures 2 and 4), the 3D form came about naturally when inserting the device into an agar phantom. The insulating polyimide layers around the $\mu \mathrm{ECoG}$ arrays had a total thickness of approximately $8 \mu \mathrm{m}$ allowing them to be ultra-flexible and conform to the surface of the brain. The addition of a $30 \mu \mathrm{m}$ thick polyimide layer (that appears dark in appearance in Figures 2D, 4A \& B) over the penetrating array, increased the stiffness of the penetrating portion of the device, allowing it to penetrate both the cortical tissue and dura. PEDOT-PSS-CNT composite was successfully polymerized on the $30 \mu \mathrm{m}$ diameter microelectrodes with very high localized precision, as can be seen from a representative picture of a PEDOT-PSSCNT coated GC microelectrodes (Figure 5B). A High-resolution SEM image of the PEDOT-PSSCNT layer shown in Figure 5C demonstrates a very porous morphology from which emerges a fine nanoscale CNT scaffold structure, similar to what was reported in a previous study on flat and spherical Au electrode surfaces [53-54]. As expected, after the deposition of the PEDOT-PSS-CNT composite, there was a significant reduction in the impedance (nearly three orders of magnitude at 1 $\mathrm{kHz}$ ) of the electrodes (Figure $6 \mathrm{~A}$ ), from $555.1 \pm 128.1 \mathrm{k} \Omega$ to $3.6 \pm 1.2 \mathrm{k} \Omega$ at $1 \mathrm{kHz}$. The impedance of the PEDOT-PSS-CNT coated $\mu$ ECoG GC microelectrodes before and after folding remain constant $(4.2 \pm 0.7 \mathrm{k} \Omega$ at $1 \mathrm{kHz})$. This indicates that the folding of the device does not damage the gold traces or the GC electrodes. EIS plots are reported in Supporting Figure S3 of Supplementary Information. The PEDOT-PSS-CNT coated GC microelectrodes present $\mathrm{CTC}_{\text {tot }}$ of $300 \mathrm{mC} / \mathrm{cm}^{2}$ in the $-0.5 / 0.5 \mathrm{~V}$ $\mathrm{EW}$ and of $458 \mathrm{mC} / \mathrm{cm}^{2}$ the $-0.6 / 0.8 \mathrm{~V} \mathrm{EW}$. The experimentally verified charge injection limit (Qinj) is $7.2 \mathrm{mC} / \mathrm{cm}^{2}$. These results are comparable to previously reported CTCs and charge 
injection limit of PEDOT-PSS-CNT coated nanostructured gold microelectrodes [54-55]. An example of CVs of PEDOT-PSS-CNT coated GC microelectrodes with two different electrochemical windows (EW) is shown in Supporting Figure S2 of Supplementary Information.

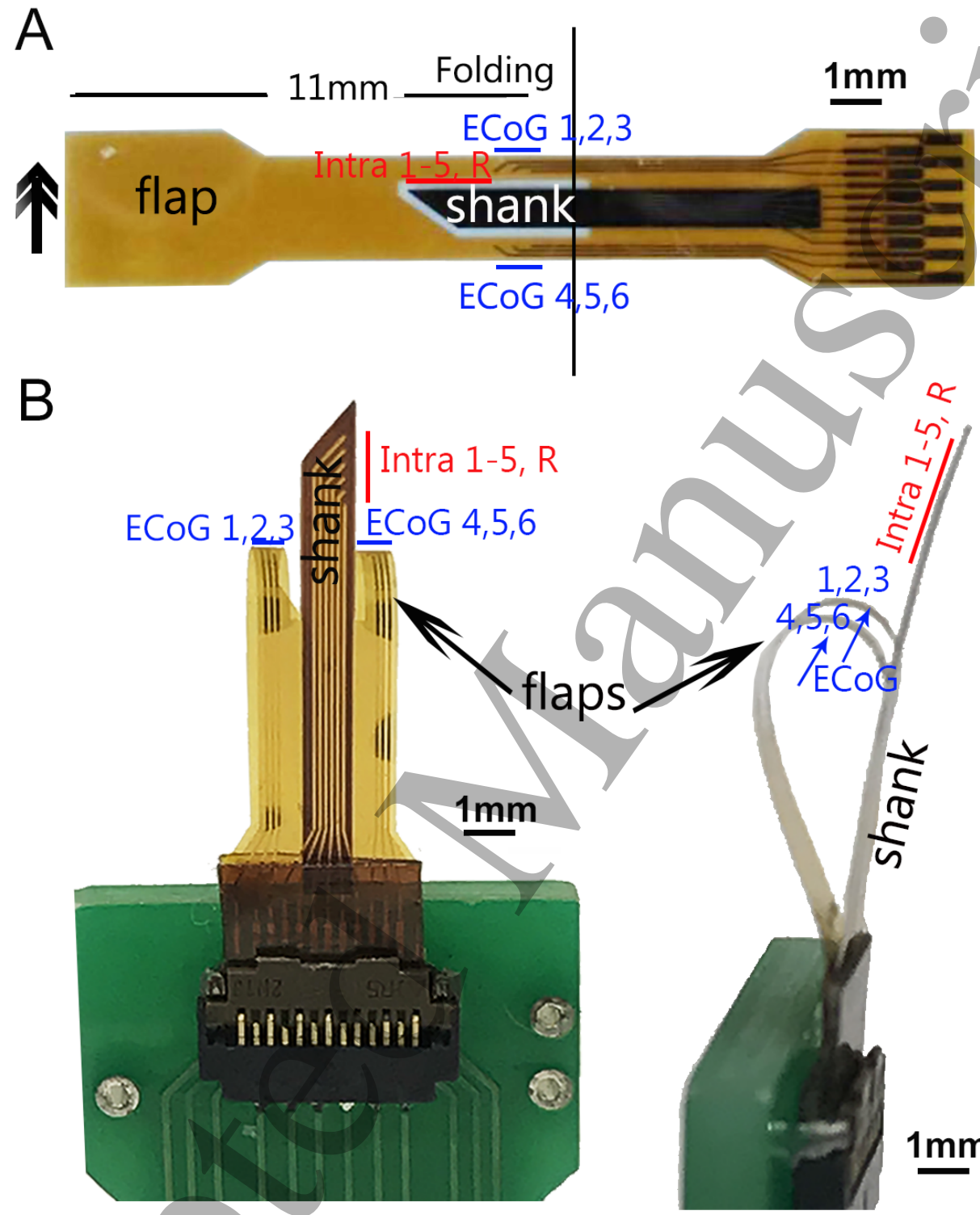

Figure 4. (A) Single-probe origami (Epi-Intra) device after being released from the carrying wafer in its 2D form; (B) Representative images of the Epi-Intra folded device assembled into the connecting PCB in its 3D conformation. 


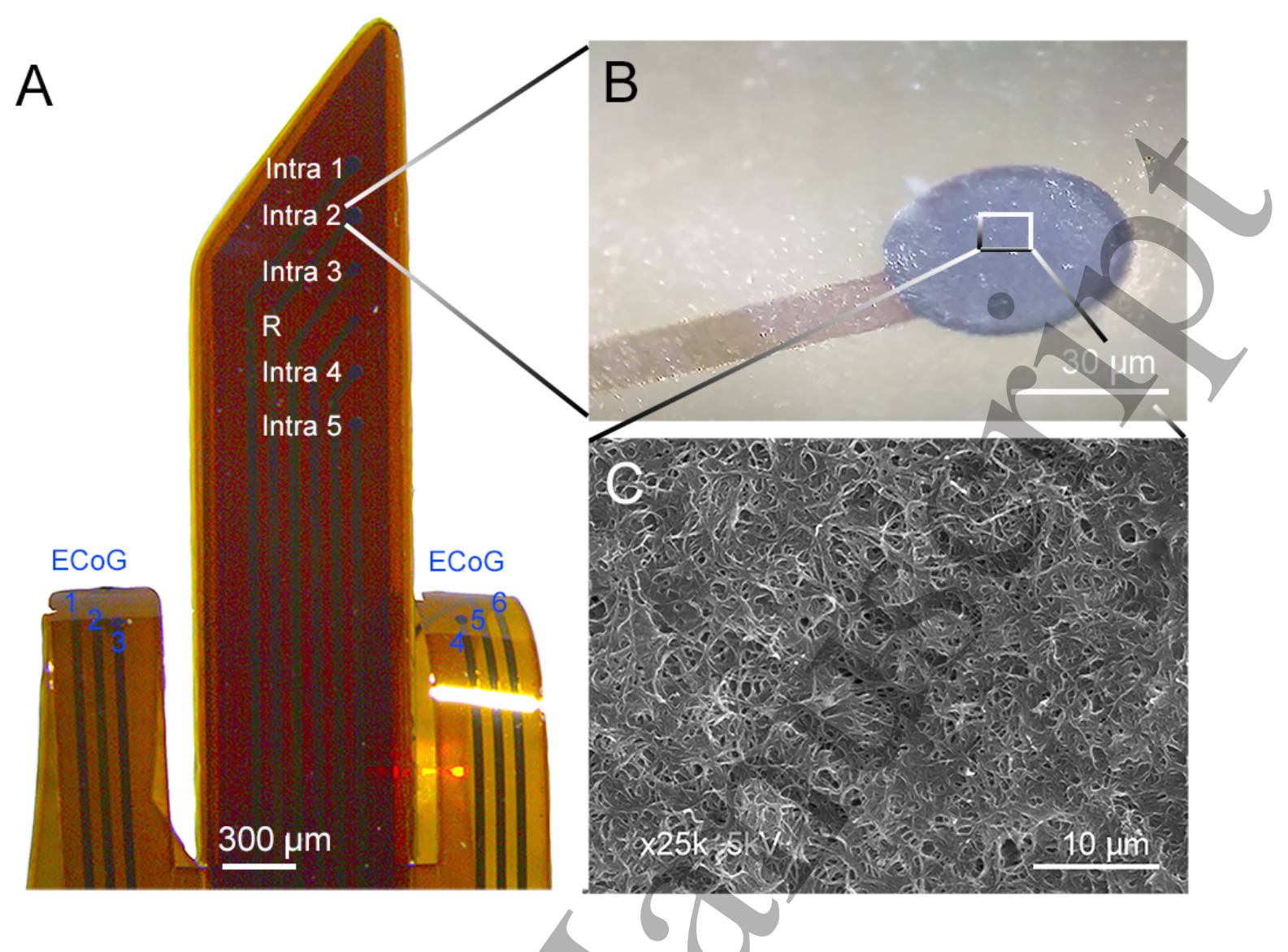

Figure 5. A. Magnification of Epi-Intra Device, B. Magnification of a PEDOT-PSS-CNT coated microelectrodes; C. SEM image of PEDOT-PSS-CNT morphology.

To validate its ability to penetrate the brain tissue and its functionality, the combined MEA was tested in vivo through acute intracortical and epicortical recording sessions in a rat model. The shaft of the device was able to penetrate the rat brain tissue and the two $\mu \mathrm{ECoG}$ array areas adhered well to the brain surface without interfering with the intracortical part. After implanting the device, we did not observe significant change in impedance of the microelectrodes, which remained nearly identical to the impedance measured during preliminary in vitro tests, for both the intracortical and epicortical recording sites $(3.6 \pm 1.2 \mathrm{k} \Omega$ at $1 \mathrm{kHz}$ before and $3.7 \pm 1.3 \mathrm{k} \Omega$ at $1 \mathrm{kHz}$ after). The impedance spectra of a representative PEDOT- PSS-CNT-coated electrode before and after an in vivo intracortical recording session are shown in Figure 6B. 

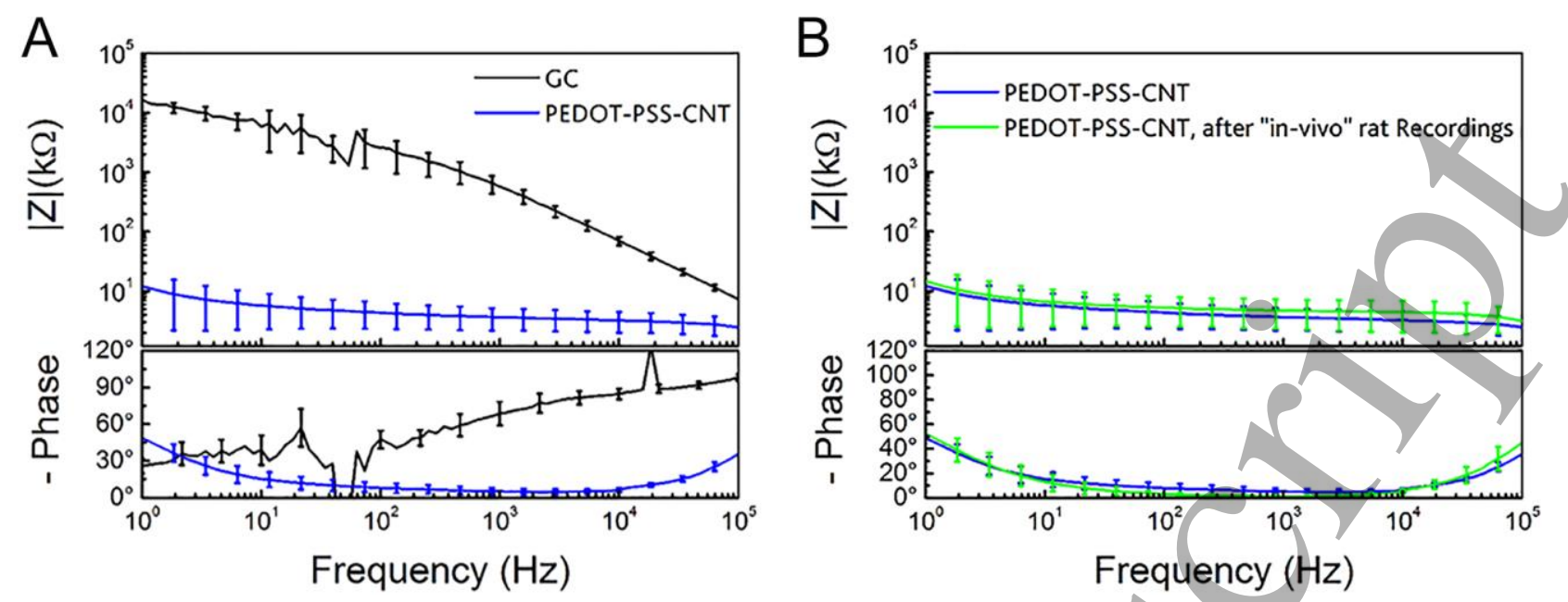

Figure 6. A. Impedance spectra (mean and deviation, $\mathrm{n}=12$ ) of the $30 \mu \mathrm{m}$ diameter microelectrodes before (black) and after PEDOT-PSS-CNT (blue) deposition; B. Impedance spectra (mean and deviation, n=12) of the 30 $\mu \mathrm{m}$ diameter PEDOT-PSS-CNT coated microelectrodes before (blue) and after (green) intra- and epicortical in vivo recordings in rat brain.

In vivo results are schematically summarized in Figure 7 . The surface electrodes were able to record typical SEPs with amplitudes following those of whiskers deflection [56] (see Table 2). For the penetrating electrodes, the amplitude of recorded high frequency signals depends on their position, with smaller values detected by electrodes nearest to the surface and larger ones by the deeper electrodes, both for stimulated and spontaneous activity (see Table 3 and Figure 7). A similar trend was found for the mean value of SNR calculated for each channel both for spontaneous and for stimulated activity, as shown in Figure S3 (Supporting Information).

\begin{tabular}{|c|c|c|c|}
\hline \multicolumn{4}{|c|}{ Surface Electrode (ECoG 1-6) } \\
\hline $\begin{array}{l}\text { Whiskers deflections } \\
(\mu \mathrm{m})\end{array}$ & 250 & 500 & 800 \\
\hline Peak-to-peak amplitude & $258.61 \pm 52.73$ & $248.53 \pm 56.81$ & $273.25 \pm 48.85$ \\
\hline
\end{tabular}

Table 2: mean SEP amplitudes for 8 acquired traces under the 3 different whiskers stimulations (mean value $\mu \mathrm{V} \pm \mathrm{SD}$ ) 


\section{Penetrating electrodes}

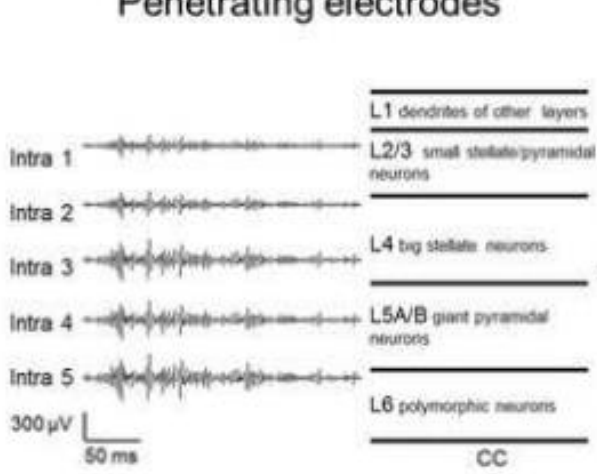

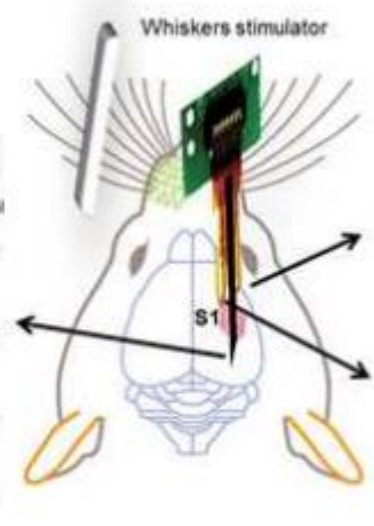

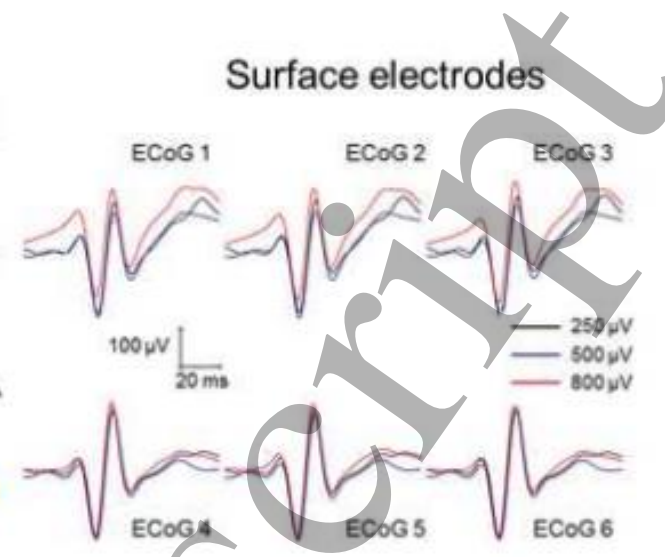

Figure 7. Schematic representation of a recording session on rat vibrissae region of $\mathrm{S} 1$. The whiskers pad, contralateral to the recorded cortex, was in contact with a flag to produce whiskers deflection at 3 different amplitudes (whiskers stimulator). The shaft of the device was inserted into the cortex perpendicularly to the surface, since each electrode was able to record neural signal. On the left side of the picture is shown an example of recorded traces from the 5 penetrating electrodes, from the most superficial layer with small signals (Intra 1,2), to the deepest (Intra 3,4,5) with large signal (scale bar $300 \mu \mathrm{V}$ and $50 \mathrm{~ms}$ ). In the figure, each trace was associated with the respective cortical layers and neuron populations during recording session. The six surface channels (ECoG 1-6) were coupled with the brain surface to record SEPs. In the right side, the small panels show an example of mean SEPs recorded after 20 cycles for each of the 3 amplitude deflections (scale bar100 $\mu \mathrm{V}$ and $20 \mathrm{~ms}$ ). In black the SEPs elicited using a $250 \mu \mathrm{m}$ whiskers deflection, in blue $500 \mu \mathrm{m}$ and in red $800 \mu \mathrm{m}$.

\begin{tabular}{|l|l|l|l|l|l|}
\hline Signal Amplitudes & Intra 1 & Intra 2 & Intra 3 & Intra 4 & Intra 5 \\
\hline Stimulated activity & $851.9 \pm 153.7$ & $871 \pm 159.6$ & $995.1 \pm 280.1$ & $1007.6 \pm 264.4$ & $1097.7 \pm 421.7$ \\
\hline Spontaneous activity & $458.7 \pm 92.7$ & $524.3 \pm 138.9$ & $557.0 \pm 231.7$ & $540.6 \pm 208.4$ & $557.0 \pm 277.9$ \\
\hline
\end{tabular}

Table 3: Signal amplitudes for each 'intra'-cortical channel following eight whiskers stimulations and two recordings from spontaneous activity (mean value $\mu \mathrm{V} \pm \mathrm{SD}$ ). 


\section{Discussion}

The orgami-style 3D platform presented here, to the best of our knowledge, is the first combined epicortical and intracortical recording platform in which the electrode material, size and fabrication method are identical between the penetrating and surface electrodes. A challenge in creating such a hybrid device stems from the fact that the ideal mechanical properties for penetrating and $\mu \mathrm{ECoG}$ arrays are different, most notably penetrating devices require a stiffer substrate to penetrate the tissue, while $\mu \mathrm{ECoG}$ arrays need to remain ultra-flexible to better conform to the brain surface. Therefore, for many combined epicortical and intracortical recording set-ups, the penetrating and $\mu \mathrm{ECoG}$ recording arrays are fabricated using different materials and methods, and as a result the electrode material and geometries are not consistent between the epi and intracortical arrays [30-32, 34-35]. This makes direct comparison between the acquired signals more difficult as the differences in the electrodes must be taken into account. Our platform employed the same fabrication method for both the epicortical and intracortical arrays, and therefore the electrodes were consistent across both portions of the device. Polyimide was chosen as the substrate material due to its mechanical and insulating properties and its ability to be deposited at significantly different thicknesses based on its initial viscosity and spin-coating speed. By varying the thickness of the polyimide layers over the $\mu \mathrm{ECoG}$ and penetrating arrays, we were able to tune the mechanical properties of the different portions of the device. Particularly, polymide HD-4100 was used as insulator layer for the foldable segment of the device because of its excellent mechanical properties and excellent resistance to thermal and chemical extremes. Durimide 7520 was added as a thickening layer of the penetrating shank because it allows for a cured film thickness up to $50 \mu \mathrm{m}$. Additionally, it presents superior mechanical property retention after extended pressure cooker test (>1000 hour) and excellent adhesion.

As can be seen in Figures 2 and 3, we are able to fabricate a variety of geometries depending on the electrophysiological study. In the example used to validate the platform (Figure 3), the $\mu \mathrm{ECoG}$ arrays were arranged on a slight convex curve. This arrangement was beneficial in two ways: (1) it 
improved the contact between the $\mu \mathrm{ECoG}$ electrodes and cortical surface and (2) it acted like a small spring, allowing the penetrating portion of the device to be inserted at varying depths without disturbing the position of the $\mu \mathrm{ECoG}$ electrodes. Unlike other examples of combined epicortical and intracortical MEAs where the vertical distance between the penetrating and intracortical electrodes are fixed once the device is fabricated [33-35], the distance between the two sets of electrodes of this device can be altered during the implant surgery. Therefore, the reported design is less impacted by any variations between devices caused by fabrication or assembly, as the penetration depth is independent of $\mu \mathrm{ECoG}$ portion of the device and can be used for multiple experiments where different penetration depths may be required. As the platform is fabricated from the thin-film, flexible polymer polyimide, it lends itself well to chronic applications where large mechanical mismatches between the device and brain tissue may lead to chronic inflammation, the formation of a glial scar and loss of device function [6]. In addition, the fact that the epicortical and intracortical arrays are integrated on a single device with one connector makes this device well suited for chronic applications by reducing the number of connections required.

Furthermore, PEDOT-PSS-CNT coatings drastically decreased the impedance of the $30 \mu \mathrm{m}$ diameter $\mu \mathrm{ECoG}$ and intracortical/microelectrodes, guaranteeing an ideal electrode conductivity of miniaturized electrodes [32]. Both cortical and intracortical recordings would greatly benefit from reduction of the impedance of recording sites. In fact, a low impedance is fundamental to enhance the signal recording quality, reduce the background noise, and, consequently, increase the signal-tonoise ratio [55]. At the same time, a lower impedance with no dimensional increase enables the injection of relatively large capacitive currents whilst minimizing electrode degradation due to Faradaic effects, as reported in our previous study on similar PEDOT-PSS coated GC microelectrodes [8]. We used electrochemical deposition because it can be performed at room temperature and applied to an unconstraint variety of materials. We adopted GC as microelectrode material for its superior electrochemical properties and PEDOT-PSS because of its high conductivity and chemical stability. Further, a combination of GC and PEDOT-PSS offers an 
excellent adhesion better than Pt and PEDOT-PSS, as previously reported [8]. In particular, we reinforced PEDOT-PSS with CNTs because PEDOT-CNT composite outperforms plain PEDOT in terms of impedance reduction, effective surface area increase, conductivity, and mechanical stability, taking advantage of the excellent properties of CNTs [36-39]. In fact, our study confirmed that PEDOT-PSS-CNT composites remain attached to the GC microelectrodes before and after folding as well as after implantation, as supported by the impedance measurements that showed no noticeable changes (Figure 6B and Supplementary Figure S3).

In summary, the recently introduces technologies [6-8] that allow for pattern transfer and the integration of GC electrodes into thin-film polymeric substrates allow us to exploit: (1) GC as microelectrode substrate, (2) flexible polymer polyimide as substrate. This provides a great advantage even compared to the state-of-the-art silicon MEMS technology, which allows to produce smaller devices, with precise and highly reproducible mechanical and geometric characteristics, but still using metal microelectrodes and a rigid substrate.

Lastly, the in vivo test has shown that the device was able to record neural signals with a high sensitivity to the physiology of the specific cortex region where the electrodes were placed (i.e. the vibrissae region of S1). For ECoG, response to variations of whiskers deflection amplitude was excellent, while for the penetrating electrodes, waveform amplitudes of recorded high frequency are in good agreement with the anatomical characteristics of the neuronal population following the laminar structure of the primary somatosensory cortex [40-43].

\section{Conclusions}

In this study, we introduced a new microfabrication and pattern transfer technology that allows origami-style 3D glassy carbon MEMS (GC MEMS) structures supported on flexible polymeric substrates. These devices demonstrate the versatility of the technology, delivering a flexible, thinfilm multipurpose platform that contains both surface (ECoG) and penetrating (intracortical) GC MEAs on a 3D device. The device can be batch-fabricated and is easily reproducible using pattern- 
transfer techniques. The platform can be fabricated in a variety of geometries and incorporates GC microelectrodes coated with PEDOT-PSS-CNT to achieve low impedance of the miniaturized electrodes. We tested the manufactured device in vivo to confirm that the shank was able to penetrate the brain tissue without buckling and that the $\mu \mathrm{ECoG}$ arrays could adhere to the cortex without damaging it or interfering with the penetrating portion. We additionally validated the ability of the device to record neural activity from the vibrissae representation of the rat primary somatosensory cortex (S1). From the physiological point of view, the advantage of this platform is that the surface and penetrating electrode contact sites are equal in design, size and material, allowing a much more reliable comparison between signals acquired epidurally and intracortically. In addition, the compact and simple design makes it suitable for chronic implants and long-term electrophysiological studies. In conclusion, the innovative platform presented here demonstrates that there is significant potential for GC-based thin-film technology to successfully interface with the brain in different configurations simultaneously from a single flexible sheet of polymeric substrate. Furthermore, we expect foreign body response to our thin and flexible integrated device during long-term implants to be less severe than the one arising after implanting stiff penetrating probes.

Acknowledgments: The authors would like to acknowledge Alice Scarpellini of IIT (Istituto Italiano di Tecnologia) Electron Microscope Facilities for SEM support.

Funding: This work was supported by the Center for Sensorimotor Neural Engineering, a National Science Foundation Engineering Research Center [EEC-1028725].

\section{Conflict of Interest Statement}

The authors declare that the research was conducted in the absence of any commercial or financial relationships that could be construed as a potential conflict of interest.

\section{Supplementary information}




\section{References}

1. R. L. McCreery, "Advanced Carbon Electrode Materials for Molecular Electrochemistry", Chem. Rev., 2008, 108 (7), pp 2646-2687, DOI: 10.1021/cr068076m, 2008.

2. O. J. A. Schueller, S. T. Brittain, C. Marzolin, and G. M. Whitesides, "Fabrication and characterization of glassy carbon MEMS," Chem. Mater., vol. 9, pp. 1399-1406, 1997.

3. Kassegne, S., Moon, K., Martín-Ramos, P., Majzoub, M., Ozturk, G., Desai, K., Parikh, M., et al. "Organic MEMS/NEMS-based High-Efficiency 3D ITO-less Flexible Photoyoltaic Cells", JMM (Journal of Micromechanics and Microengineering), 22 (11), 115015, 2012.

4. Wang, C., Jia, G., Taherabadi, L.H., Madou, M.J., "A novel method for the fabrication of highaspect ratio C-MEMS structures", Journal of microelectromechanical systems, 2005.

5. Ranganathan, S., McCreey, R., Majji, S.M., Madou, M., "Photoresist-derived Carbon for Microelectromechanical Systems and Electrochemical Applications", J Electrochem Soc, 147 (2000), pp. 277-282

6. M. Vomero, P. van Niekerk, V. Nguyen, N. Gong, M. Hirabayashi, A. Cinopri, K. Logan, A. Moghadasi, P. Varma, S, Kassegne, A novel pattern transfer technique for mounting glassy carbon microelectrodes on polymeric flexible substrates, Journal of Micromechanics and Microengineering 2016; 26: 025018. Doi:10.1088/0960-1317/26/2/025018.

7. S. Kassegne, M. Vomero, R. Gaguglio, M. Hirabayashi, E. Özyilmaz, S. Nguyen, J. Rodriguez, E. Özyilmaz, P. van Niekerk, A. Khosla, Electrical impedance, electrochemistry, mechanical stiffness, and hardness tunability in glassy carbon MEMS $\mu E C o G$ electrodes. Microelectronic Engineering 133 (2015) 36-44. http://dx.doi.org/10.1016/j.mee.2014.11.013

8. M. Vomero, E. Castagnola, F. Ciarpella, E. Maggiolini, N. Goshi, E. Zucchini, S. Carli, L. Fadiga, S. Kassegne, D. Ricci, Highly Stable Glassy Carbon Interfaces for Long-Term Neural Stimulation and Low-Noise Recording of Brain Activity, Nature Sci. Rep 7 (2017) 40332. doi: 10.1038/srep40332.

9. S. Takeuchi, T. Suzuki, K. Mabuchi and H. Fujita 3D flexible multichannel neural probe array 
J. Micromech, Microeng. 14 (2004) 104-107.

10. J. P. Whitney, P. S. Sreetharan, K. Y. Ma, R. J. Wood, Pop-up book MEMS, Journal of Micromechanics and Microengineering 21(11) (2011): 115021.

11. S. A. Zotov, M. C. Rivers, A. A. Trusov, A. M. Shkel, Folded MEMS pyramid inertial measurement unit, IEEE Sensors Journal 11(11) (2011) 2780-2789.

12. E. E. Hui, R. T. Howe, M. S.Rodgers. Single-step assembly of complex 3-D microstructures. Micro Electro Mechanical Systems, 2000. MEMS 2000. The Thirteenth Annual International Conference on, pp. 602-607.

13. A. C. Siegel, D. A. Bruzewicz, D. B. Weibel, G. M. Whitesides. Microsolidics: fabrication of three dimensional metallic microstructures in poly (dimethylsiloxane). Advanced Materials 19(5) (2007): 727-733.

14. G. Buzsáki, Large-scale recording of neuronal ensembles, Nature neuroscience 7 (2004) 446451. doi:10.1038/nn1233

15. C. Palmer, A microwire technique for recording single neurons in unrestrained animals, Brain research bulletin 3 (1978) 285-289. http://dx.doi.org/10.1016/0361-9230(78)90129-6

16. C.M. Gray, P.E. Maldonado, M. Wilson, B. McNaughton, Tetrodes markedly improve the reliability and yield of multiple single-unit isolation from multi-unit recordings in cat striate cortex, Journal of neuroscience methods 63 (1995) 43-54. http://dx.doi.org/10.1016/01650270(95)00085-2

17. P. K. Campbell, K.E. Jones, R.J. Huber, K.W. Horch, R.A. Normann, A silicon-based, threedimensional neural interface: manufacturing processes for an intracortical electrode array, IEEE Trans Biomed Eng. 38(1991) 758-768. doi: 10.1109/10.83588

18. V.S. Polikov, P.A. Tresco, W. M. Reichert, Response of brain tissue to chronically implanted neural electrodes, J Neurosci Methods. 148 (2005) 1-18. doi: 10.1016/j.jneumeth.2005.08.015

19. E.C. Leuthardt, G. Schalk, J.R. Wolpaw, J. G. Ojemann, D.W. Moran, A brain-computer interface using electrocorticographic signals in humans, J. Neural Eng. 1 (2004) 63 doi: 
$10.1088 / 1741-2560 / 1 / 2 / 001$

20. B. Rubehn, C. Bosman, R. Oostenveld , P. Fries, T. Stieglitz, A MEMS-based flexible multichannel ECoG-electrode array, J Neural Eng. 6 (2009) 036003. DOI: 10.1088/1741$2560 / 6 / 3 / 036003$

21. J. Viventi, D-H Kim, L. Vigeland, et al., Flexible, foldable, actively multiplexed, high-density electrode array for mapping brain activity in vivo, Nature neuroscience 14 (2011) 1599-1605. doi:10.1038/nn.2973

22. G. Hotson, D.P. McMullen, M. F. Fifer, M.S. Johannes, K.D. Katya1, M.P. Para, R. Armiger, W.S. Anderson, N.V. Thakor, B.A. Wester, N.E. Crone, Individual finger control of a modular prosthetic limb using high-density electrocorticography in a human subject, J. Neural Eng. 13 (2016) 026017. doi: 10.1088/1741-2560/13/2/026017

23. E. Castagnola, L. Maiolo, E. Maggiolini, A. Minotti, M. Marrani, F. Maita, A. Pecora, G.N. Angotzi, A. Ansaldo, M. Boffini, L. Fadiga, G. Fortunato, D. Ricci, PEDOT-CNT-coated lowimpedance, ultra-flexible, and brain-conformable $\mu E C o G$ arrays, IEEE Trans Neural Syst Rehabil Eng. 2015; 23: 342-350. Doi: 10.1109/TNSRE.2014.2342880

24. D. Khodagholy, T. Doublet, P/Quilichini, M. Gurfinkel, P. Leleux, A. Ghestem, E. Ismailova, T. Hervé, S. Sanaur, C. Bernard, G. G. Malliaras, In vivo recordings of brain activity using organic transistors, Nature communications 4 (2013); 4:1575. DOI: 10.1038/ncomms2573

25. D. Khodagholy, J.N. Gelinas, T. Thesen, W. Doyle, O. Devinsky, G.G. Malliaras, G. Buzsáki, NeuroGrid: recording action potentials from the surface of the brain, Nature neuroscience 18 (2015) 310-315. doi:10.1038/nn.3905.

26. G. Schalk, E.C. Leuthardt, Brain-computer interfaces using electrocorticographic signals, IEEE Rev Biomed Eng. 4 (2011) 140-154. doi: 10.1109/RBME.2011.2172408.

27. A. Ritaccio, P. Brunner, M.C. Cervenka, N. Crone, C. Guger, E. Leuthardt, R. Oostenveld, W. Stacey, G. Schalk, Proceedings of the first international workshop on advances in electrocorticography, Epilepsy \& Behavior 19 (2010) 204-215. 
http://dx.doi.org/10.1016/j.yebeh.2010.08.028

28. A. Ritaccio, P. Brunnera, A. Gunduz, D. Hermes, L. J. Hirsch, J. Jacobs, K. Kamada, S. Kastner, R. T. Knight, R. P. Lesser, et al., Proceedings of the Fifth International Workshop on Advances in Electrocorticography. Epilepsy \& Behavior 41 (2014) 183-192. http://dx.doi.org/10.1016/j.yebeh.2014.09.015

29. H. Toda, T. Suzuki, H. Sawahata, K. Majima, Y. Kamitani, I. Hasegawa, Simultaneous recording of ECoG and intracortical neuronal activity using a flexible multichannel electrodemesh in visual cortex. Neuroimage 54 (2011) 203-212. doi: 10.1016/j.neuroimage.2010.08.003.

30. H. Watanabe, T. Sakatani, T. Suzuki, M.A. Sato, Y. Nishimura, A. Nambu, M. Kawato, T. Isa, Reconstruction of intracortical whisker-evoked local field potential from electrocorticogram using a model trained for spontaneous activity in the rat barrel cortex, Neurosci Res. 87 (2014) 40-48. doi: 10.1016/j.neures.2014.06.010.

31. S.N. Baker, G. Curio, N.L. Roger, EEG oscillations at $600 \mathrm{~Hz}$ are macroscopic markers for cortical spike bursts. J Physiol. 550 (2003) 529-534. doi: 10.1113/jphysiol.2003.045674.

32. H. Watanabe, M.A. Sato, T. Suzuki, A. Nambu, Y. Nishimura, M. Kawato, T. Isa, Reconstruction of movement-related intracortical activity from micro-electrocorticogram array signals in monkey primary motor cortex. J. Neural Eng. 9 (2012) 036006. doi: 10.1088/17412560/9/3/036006.

33. G. Márton, G. Orbán, M. Kiss, R. Fiáth, A. Pongrácz, I. Ulbert, A Multimodal, SU-8-PlatinumPolyimide Microelectrode Array for Chronic In Vivo Neurophysiology, PloS One 10 (2015) e0145307. http://dx.doi.org/10.1371/journal.pone.0145307.

34. Z. Xiang, S.C. Yen, S. Sheshadri, N. Xue, S.H. Lee, J. Wang, N.V. Thakor, C. Lee, Flexible and self-adaptive neural ribbon with three-dimensional electrodes for sciatic nerve recording. Conf Proc IEEE Eng Med Biol Soc. 2015; 3157-3160

35. Z. Xiang, J. Liu, C. Lee, A flexible three-dimensional electrode mesh: An enabling technology for wireless brain-computer interface prostheses, Microsystems \& Nanoengineering 2 (2016) 
16012. doi:10.1038/micronano.2016.12

36. R.A. Green, C.M. Williams, N.H. Lovell, L. A. Poole-Warren, Novel neural interface for implant electrodes: Improving electroactivity of polypyrrole through MWCNT incorporation, J MATER SCI-MATER M 19 (2008) 1625-1629. doi:10.1007/s10856-008-3376-7

37. R. Gerwig, K. Fuchsberger, B. Schroeppel, G. S. Link, G. Heusel, U. Kraushaar, W. Schuhmann, A. Stett, M. Stelzle, PEDOT-CNT composite microelectrodes for recording and electrostimulation applications: fabrication, morphology, and electrical properties, Front. Neuroeng. 5 (2012) 8. doi: 10.3389/fneng.2012.00008

38. E. Castagnola, L. Maiolo, E. Maggiolini, A. Minotti, M. Marrani,,F. Maita, A. Pecora, G. N. Angotzi, A. Ansaldo, L. Fadiga, G. Fortunato, D. Ricci, Ultra-flexible and brain-conformable microelectrocorticography device with low impedance PEDOT-carbon nanotube coated microelectrodes, Neural Engineering (NER), 2013 6th International IEEE/EMBS Conference on, San Diego, California, 6 - 8 November 2013; 927-930. doi: 10.1109/NER.2013.6696087

39. S. Chen, W. Pei, Q. Gui, R. Tang, Y. Chen, S. Zhao, H. Wang, H. Chen, PEDOT/MWCNT composite film coated microelectrode arrays for neural interface improvement, Sens. Actuator A-Phys. 193 (2013) 141-148. http://dx.doi.org/10.1016/j.sna.2013.01.033

40. J.K. Chapin. Laminar differences in sizes, shapes and response profiles of cutaneous receptive fields in the rat S1 cortex. Exp. Brain Res. 62 (1986) 549-559. doi: 10.1007/BF00236033

41. D.J. Simons, Response properties of vibrissa units in rat SI somatosensory neocortex, J. Neurophysiol. 41 (1978) 798-820.

42. C.C.H. Petersen, The Functional Organization of the Barrel Cortex, Neuron 56 (2007) 339-355. http://dx.doi.org/10.1016/j.neuron.2007.09.017

43. G.E. Carvell, D.J.Simons, Membrane potential changes in rat SmI cortical neurons evoked by controlled stimulation of mystacial vibrissae, Brain Research 448 (1998) 186-191. http://dx.doi.org/10.1016/0006-8993(88)91118-3

44. J. Winnie, K. Yoshida, and U. G. Hofmann. In-vivo implant mechanics of flexible, silicon-based 
ACREO microelectrode arrays in rat cerebral cortex. IEEE Transactions on Biomedical Engineering 53(5) (2006): 934-940.

45. Fujifilm. "Durimide ${ }^{\circledR} 7500$ Photosensitive Polyimide Precoursor.” Technical Product Information. Revised May, 2012.

46. M. Jeon, J. Cho, Y. K. Kim, D. Jung, E.-S. Yoon, S. Shin, Il-J. Cho, Partially flexible MEMS neural probe composed of polyimide and sucrose gel for reducing brain damage during and after implantation, Journal of Micromechanics and Microengineering 24(2): 025010, 2014

47. T. Stieglitz, H. Beutel, M. Schuettler, J.-U. Meyer, Micromachined, Polyimide-Based Devices for Flexible Neural Interfaces, Biomedical Microdevices, 2(4), pp 283-294 (2000)

48. S. Bossi, S. Kammer, T. Doerge, A. Menciassi, K. P. Hoffmann, S. Micera. Prototype development of an actuated intraneural electrode, IFESS 2008-From Movement to Mind, 13th Annual International FES Society. Conference. Friburgo, Germania, 21 - 25 settembre, 2008

49. X. Jiang, X. Sui, Y. Lu, Y. Yan, C. Zhou, L. Li, Q. Ren and X. Chai, In vitro and in vivo evaluation of a photosensitive polyimide thin-film microelectrode array suitable for epiretinal stimulation, Journal of NeuroEngineering and Rehabilitation 2013, 10:48

50. S. P. Lacour, R. Atta, J.J. FitzGeraldc, M. Blamire, E. Tarte, J. Fawcett, Polyimide microchannel arrays for peripheral nerve regenerative implants, Sensors and Actuators A: Physical, 147 (2008) 456-463

51. Y. Sun,S. P. Lacour,R. A. Brooks, N. Rushton, J. Fawcett, R. E. Cameron, Assessment of the biocompatibility of photosensitive polyimide for implantable medical device use, J Biomed Mater Res A. 90(3):648-55 2009,

52. G. Paxinos, C. Watson. The Rat Brain in Stereotaxic Coordinates, 6th Edn. Amsterdam: Elsevier (2007)

53. E. Castagnola, A. Ansaldo, E. Maggiolini, G.N. Angotzi, M. Skrap, D. Ricci, L. Fadiga, Biologically compatible neural interface to safely couple nanocoated electrodes to the surface of the brain, ACS Nano 7 (2013) 3887-3895. doi: 10.1021/nn305164c 
54. E. Castagnola, E. Maggiolini, L. Ceseracciu, F.Ciarpella, E. Zucchini, S. De Faveri, L. Fadiga, D. Ricci, pHEMA Encapsulated PEDOT-PSS-CNT Microsphere Microelectrodes for Recording Single Unit Activity in the Brain. Front Neurosci.10 (2016) 151. doi:10.3389/fnins.2016.00151

55. N. Mesgarani, C. Cheung, K. Johnson, E. F. Chang, Phonetic Feature Encoding in Human Superior Temporal Gyrus, Science 343 (2014) 1006-1010. DOI: 10.1126/science.1245994

56. E. Castagnola, A. Ansaldo, E. Maggiolini,T. Ius, M. Skrap, D. Ricci, L. Fadiga, Smaller, softer, lower-impedance electrodes for human neuroprosthesis: a pragmatic approach, Front Neuroeng 7 (2014). Doi: 10.3389/fneng.2014.00008. 